\title{
Can Myogenic Tone Protect Endothelial Function? Integrating Myogenic Activation and Dilator Reactivity for Cerebral Resistance Arteries in Metabolic Disease
}

\author{
Brayden D. Halvorson ${ }^{a}$ John J. McGuire ${ }^{a}$ Krishna K. Singh ${ }^{a}$ Joshua T. Butcher ${ }^{b}$ \\ Julian H. Lombard ${ }^{c}$ Paul D. Chantler ${ }^{d}$ Jefferson C. Frisbee ${ }^{a}$ \\ aDepartments of Medical Biophysics, Schulich School of Medicine and Dentistry, University of Western Ontario, \\ London, ON, Canada; ' Department of Physiological Sciences, Oklahoma State University, Stillwater, OK, USA;

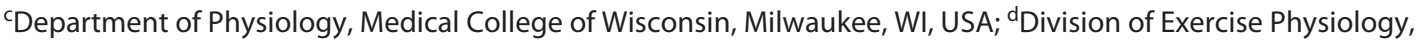 \\ West Virginia University Health Sciences Center, Morgantown, WV, USA
}

\section{Keywords}

Microcirculation - Rodent models of metabolic disease .

Cerebral circulation - Regulation of vascular tone

\begin{abstract}
The obese Zucker rat (OZR) manifests multiple risk factors for impaired cerebrovascular function, including hypertension and insulin resistance although how they combine to produce integrated vascular function is unclear. As studies have suggested that myogenic activation (MA) severity for middle cerebral arteries (MCAs) may be proportional to hypertension severity, we hypothesized that MA will negatively correlate with dilator reactivity in OZR. MA of MCA from OZR was divided into low, medium, and high based on the slope of MA, while MCA reactivity and vascular metabolite bioavailability were assessed in all groups. Endothelium-dependent dilation of MCA in OZR was attenuated and correlated with the MA slope. Treatment of OZR MCA with TEMPOL (antioxidant) improved dilation in low or medium MA groups, but had less impact on high MA. Alternatively, treatment
\end{abstract}

with gadolinium to normalize MA in OZR had reduced impact on dilator reactivity in MCA from low and medium MA groups, but improved responses in the high group. Treatment with both agents resulted in dilator responses that were comparable across all groups. These results suggest that, under conditions with stronger MA, endothelial function may receive some protection despite the environment, potentially from the ability of MCA to reduce wall tension despite increased pressure.

(c) 2021 S. Karger AG, Basel

\section{Introduction}

The term "metabolic syndrome" is used to define a cluster of risk factors for elevated cerebro- or cardiovascular disease risk, which can include obesity, hypertension, atherogenic dyslipidemia, and impaired glycemic control [1]. The prevalence of these pathologies, both individually and in combination, is growing in Western society and has become an increasing global health concern 
with the ongoing development and modernization of economies [2-4]. Individually, each of the risk factors comprising the metabolic syndrome has been implicated in the increased risk for development of cerebro- and cardiovascular dysfunction but, when presented concurrently, they greatly increase the risk beyond that of any single constituent [5]. As seen in stroke or transient ischemic attack, these vascular complications can be especially detrimental when they affect the cerebral circulation due to the potentially devastating consequences on cognition and behavior, as well as both the overall quality of life and mortality [6-8]. Apart from the decreased life expectancy and lower quality of life for individuals with metabolic diseases, there is also a significant economic cost to society as a result of this multi-pathology condition as the average annual total health care costs were found to increase by an average of $24 \%$ per additional risk factor associated with metabolic diseases [7]. These costs are further enlarged when taking into account the indirect effects associated with lost economic productivity.

Within the cerebral resistance circulation, the occurrence of increased myogenic activation (MA) and endothelial dysfunction with the evolution of the metabolic syndrome takes on a central importance as these outcomes can lead to a condition where vasoconstrictor tone can predominate to a greater than desired degree, resulting in an increased resistance for blood flow to the brain and for perfusion within cerebral tissue. However, beyond a global concept of multiple risk factors combining to negatively impact indices of cerebrovascular reactivity, it is challenging to identify how specific risk factors contribute to the well-documented alterations to vascular responses. It may be instructive to note that within models of type II diabetes mellitus in the absence of hypertension, MA is largely normal, despite significant impairments to normal endothelial function [8]. Although the magnitude of elevated arterial pressure can be highly variable, it has also been previously identified that the slope of MA of resistance vessels can be reasonably predicted by the severity of the developed hypertension in animal models [9, 10]. Further, in the human population, a reduction in blood pressure $(5 \mathrm{~mm} \mathrm{Hg}$ in diastolic blood pressure or $10 \mathrm{~mm} \mathrm{Hg}$ in systolic blood pressure) has been shown to reduce stroke risk by $30-40 \%$, clearly emphasizing the key role of blood pressure regulation in cerebrovascular health [11]. Given the central role of MA in the ongoing regulation of cerebral blood flow and perfusion distribution, a clearer understanding of how alterations to MA in the presence of hypertensive metabolic disease impacts integrated vascular reactivity is critical.

Metabolic Syndrome and Cerebral Vasculopathy
The obese Zucker rat (OZR) model of metabolic syndrome $[4,5,12]$ can be a useful tool for addressing this issue as the level of hypertension that develops in this model is both heterogenous and predictive of the degree of MA within cerebral resistance arteries $[13,14]$. The characteristics of OZR, including comments on its utility as a translationally relevant model of elevated cardio- and cerebrovascular disease risk, have been extensively reviewed elsewhere [15]. With the development of a diverse degree of hypertension in a setting of relatively severe, and consistent, insulin resistance, the OZR may provide a useful tool for investigation into how MA can impact integrated vascular reactivity within the cerebral resistance arteries. As such, the purpose of this study was to determine how differences in the severity of altered MA impact the reactivity of middle cerebral arteries (MCAs) of OZR. It was hypothesized that the degree of MA will negatively correlate with dilator reactivity in the pro-inflammatory, pro-oxidant environment of OZR.

\section{Materials and Methods}

\section{Animals}

Male lean Zucker rats (LZRs; total $n=20$; Harlan/Envigo) and OZR (total $n=60$; Harlan/Envigo) were purchased at $\sim 9$ weeks of age and were maintained on standard chow and tap water ad libitum until the time of terminal experiments. Animals were housed in animal care facilities at the Medical College of Wisconsin, the West Virginia University Health Sciences Center, or the University of Western Ontario until $\sim 17$ weeks of age, and all protocols received prior IACUC approval. At $\sim 17$ weeks of age, each rat was anesthetized with sodium pentobarbital (50 mg/ $\mathrm{kg}^{-1}$ i.p.), and the trachea was intubated to maintain a patent airway. In all rats, a carotid artery and an external jugular vein were cannulated to measure arterial pressure and to infuse additional anesthetic, respectively, as necessary. Prior to euthanasia, an aliquot of blood was drawn from the jugular vein of each animal to be used for the subsequent determination of plasma metabolic/endocrine, oxidant stress, and inflammatory biomarker profiles using commercially available kits (Millipore).

While deeply anesthetized, each rat was decapitated, after which the brain was removed from the skull case and placed in cold physiological salt solution (PSS; $4^{\circ} \mathrm{C}$ ). Subsequently, the MCAs were dissected from their origin at the circle of Willis. Both MCAs were doubly cannulated in a heated chamber $\left(37^{\circ} \mathrm{C}\right)$ that allowed the lumen and exterior of the vessel to be perfused and superfused, respectively, with PSS from separate reservoirs. The PSS was equilibrated with a $21 \% \mathrm{O}_{2}, 5 \% \mathrm{CO}_{2}$, and $74 \% \mathrm{~N}_{2}$ gas mixture and had the following composition (mM): $119 \mathrm{NaCl}, 4.7 \mathrm{KCl}, 1.17 \mathrm{MgSO}_{4}$, $1.6 \mathrm{CaCl}_{2}, 1.18 \mathrm{NaH}_{2} \mathrm{PO}_{4}, 24 \mathrm{NaHCO}_{3}, 0.026 \mathrm{EDTA}$, and 5.5 glucose. Any side branches were ligated using a single strand teased from 6-0 suture. Vessel diameter was measured using television microscopy and an on-screen video micrometer [8].

Immediately following decapitation, the thoracic aorta and multiple conduit arteries (carotid, femoral, iliac, etc.) were rapidly

J Vasc Res 2021;58:286-300 
surgically removed from the animal, rinsed in PSS to remove any remaining blood, and placed in chilled PSS prior to use in the determination of nitric oxide (NO) and $\mathrm{H}_{2} \mathrm{O}_{2}$ bioavailability (described below). In addition, following the removal of the MCA from the brain, the circle of Willis and all remaining arterial branches arising from that were surgically dissected and stored under chilled PSS prior to the determination of arachidonic acid metabolite production (described below).

\section{Measurements of Vascular Reactivity in Isolated MCA}

Following cannulation, MCAs were extended to their in situ length and equilibrated at $80 \%$ of the animal's mean arterial pressure ( $88 \pm 3 \mathrm{~mm} \mathrm{Hg}$ for LZR; $109 \pm 4 \mathrm{~mm} \mathrm{Hg}$ for OZR) to approximate in vivo perfusion pressure [16]. Any vessel that did not demonstrate substantial active tone $(<25 \%)$ at the equilibration pressure was discarded. Active tone at the equilibration pressure was calculated as $\left(\Delta D / D_{\max }\right) 100$, where $\Delta D$ is the diameter increase from rest in response to $\mathrm{Ca}^{2+}$-free PSS, and $\mathrm{D}_{\max }$ is the maximum diameter measured at the equilibration pressure in $\mathrm{Ca}^{2+}$-free PSS. Active tone for vessels in the present study averaged $37 \pm 3 \%$ in LZR and $36 \pm 3 \%$ in OZR.

Following equilibration, MA was assessed in MCAs over the range of $20-160 \mathrm{~mm} \mathrm{Hg}$ in $20 \mathrm{~mm} \mathrm{Hg}$ increments. Pressure was changed non-sequentially, and vessels were allowed $10 \mathrm{~min}$ to equilibrate at each pressure before the inner and outer diameters of the MCA were recorded. Vascular reactivity curves in each vessel were then determined in response to increasing concentrations of acetylcholine $\left(10^{-10}-10^{-6} \mathrm{M}\right)$, bradykinin $\left(10^{-11}-10^{-7} \mathrm{M}\right)$, or adenosine $\left(10^{-10}-10^{-6} \mathrm{M}\right)$. In addition, vessels were exposed to hypoxia (reduced $\mathrm{PO}_{2}$ in the vessel chamber from $\sim 130$ to $\sim 40 \mathrm{~mm}$ $\mathrm{Hg})[16]$.

Vascular responses to all stimuli were also performed following acute incubation (45-60 min) with TEMPOL, gadolinium, or both. TEMPOL $\left(10^{-4} \mathrm{M}\right)$ is a cell permeable superoxide dismutase mimetic that lowers reactive oxidant stress and provides insight into the impact of endothelial dysfunction in general [17]. Further, when combined with supplemental measurements, TEMPOL can also allow for insights into the roles of endothelial production of $\mathrm{NO}$, hydrogen peroxide $\left(\mathrm{H}_{2} \mathrm{O}_{2}\right)$, and metabolites of arachidonic acid (prostacyclin, $\mathrm{PGI}_{2}$; thromboxane, $\mathrm{TxA}_{2}$ ) and oxidative stress, respectively, in modulating vascular reactivity. Gadolinium is a blocker of mechano-sensitive stretch-activated cation channels [18-20]. Gadolinium has been shown to attenuate myogenic constriction in response to increased intralumenal pressure [18-21] and is widely used in isolated vessel preparations to inhibit mechanosensitive cation channels, thus limiting myogenic constriction. By eliminating the difference in MA between groups in the present study, gadolinium allows for better investigation of endothelial function because the propensity to dilate when challenged with endothelial dependent dilators is not being blunted by the enhanced myogenic tone. In a pilot subset of MCA, following determination of the slope of MA, individual vessels were treated with low-dose gadolinium $\left(10^{-9}-10^{-7} \mathrm{M}\right)$ to blunt the enhanced MA to levels that were comparable to that determined in MCA from control LZR (data not shown).

\section{Measurement of Vascular $\mathrm{NO}$ and $\mathrm{H}_{2} \mathrm{O}_{2}$ Bioavailability}

From each rat, the thoracic aorta and multiple conduit arteries were removed for the assessment of vascular $\mathrm{NO}$ and $\mathrm{H}_{2} \mathrm{O}_{2}$ production using amperometric sensors (World Precision Instru- ments, Sarasota, FL, USA). Briefly, arteries were isolated and pooled in a sealed chamber filled with warmed $\left(37^{\circ} \mathrm{C}\right)$ PSS equilibrated with $95 \% \mathrm{O}_{2}$ and $5 \% \mathrm{CO}_{2}$ that allowed for insertion of individual sensors. A NO sensor (ISO-NOPF 100) and an $\mathrm{H}_{2} \mathrm{O}_{2}$ sensor (ISO-HPO-100) were placed in close apposition to the pooled vessels and a baseline level of current was obtained. Subsequently, increasing concentrations of methacholine $\left(10^{-10}-10^{-6} \mathrm{M}\right)$ were added to the chamber and the changes in current were determined.

\section{Determination of Vascular Metabolites of Arachidonic Acid}

Vascular production of 6-keto-prostaglandin $\mathrm{F}_{1 a}$ (6-keto$\mathrm{PGF}_{1 \alpha}$; the stable breakdown product of $\mathrm{PGI}_{2}$ ) and 11-dehydrothromboxane $\mathrm{B}_{2}$ (11-dehydro- $\mathrm{TxB}_{2}$; the stable plasma breakdown product of $\mathrm{TxA}_{2}$ ) in response to challenge with $10^{-6} \mathrm{M}$ arachidonic acid was assessed using all other cerebral resistance arteries (e.g., circle of Willis, anterior and posterior cerebral arteries, basilar artery) as well as pooled conduit arteries (femoral, saphenous, iliac) from each rat. Pooled arteries from each animal were incubated in microcentrifuge tubes in $1 \mathrm{~mL}$ of PSS for 30 min under control conditions. After this time, the superfusate was removed, stored in a new microcentrifuge tube, and frozen in liquid $\mathrm{N}_{2}$, while a new aliquot of PSS was added to the vessels and the arachidonic acid challenge was imposed for the subsequent $30 \mathrm{~min}$. After the second 30-min period, this new PSS was transferred to a fresh tube, frozen in liquid $\mathrm{N}_{2}$, and stored at $-80^{\circ} \mathrm{C}$ until final processing. Vessels were removed and blotted to remove all excess moisture, and the total mass of the tissue was determined. $\mathrm{Me}$ tabolite release by the vessels was determined using commercially available EIA kits for 6-keto-PGF $1 \alpha$ and 11-dehydro- $\mathrm{TxB}_{2}$ (Cayman). In pilot studies, there was no discernible production of 6-keto-PGF ${ }_{1 \alpha}$ or 11-dehydro- $\mathrm{TxB}_{2}$ using the EIA kits in the absence of the vascular tissue.

\section{Data and Statistical Analyses}

Using historical data collected previously with OZR and the control LZR at $\sim 17$ weeks of age $[13,17,21,22]$, a scatter plot was developed that compared mean arterial pressure with the slope $(\beta$; calculated from linear regression analyses) of MA for ex vivo MCAs. Figure la presents the results of this comparison and suggests that not only is the level of elevated mean arterial pressure a robust predictor of the increased slope of MA, but that changes in myogenic slope present a distributed range from "no change" to a maximum of $\sim 0.38 \mu \mathrm{m} / \mathrm{mm} \mathrm{Hg}$. Based on this, and the fact that we had no a priori reason for any other classification, the myogenic slopes of MCA from animals used in the current study were placed into 1 of the 3 categories:

1. "Low" ( $n=20$ OZR), spanning slopes of $<-0.12 \mu \mathrm{m} / \mathrm{mm} \mathrm{Hg}$.

2. "Medium" ( $n=20$ OZR), spanning slopes of $-0.13 \mu \mathrm{m} / \mathrm{mm} \mathrm{Hg}$ to $-0.24 \mu \mathrm{m} / \mathrm{mm} \mathrm{Hg}$.

3. "High" ( $n=20$ OZR), spanning slopes of $>0.24 \mu \mathrm{m} / \mathrm{mm} \mathrm{Hg}$.

Figure $1 \mathrm{~b}$ presents the scatter plot of the slope of MCA MA and measured mean arterial pressure in all animals in the present study. These data reproduce the relationship between MAP and MA of MCA, suggested from the historical data in Figure 1a. Table 1 also presents the baseline data from OZR in the present study following division of MA into the 3 groups: "low," "medium," and "high." When separated in this manner, there was minimal difference between groups for all indices except mean arterial pressure, which demonstrated a consistent increase across groups, reaching statistical significance between the "low" and "high" groups. 


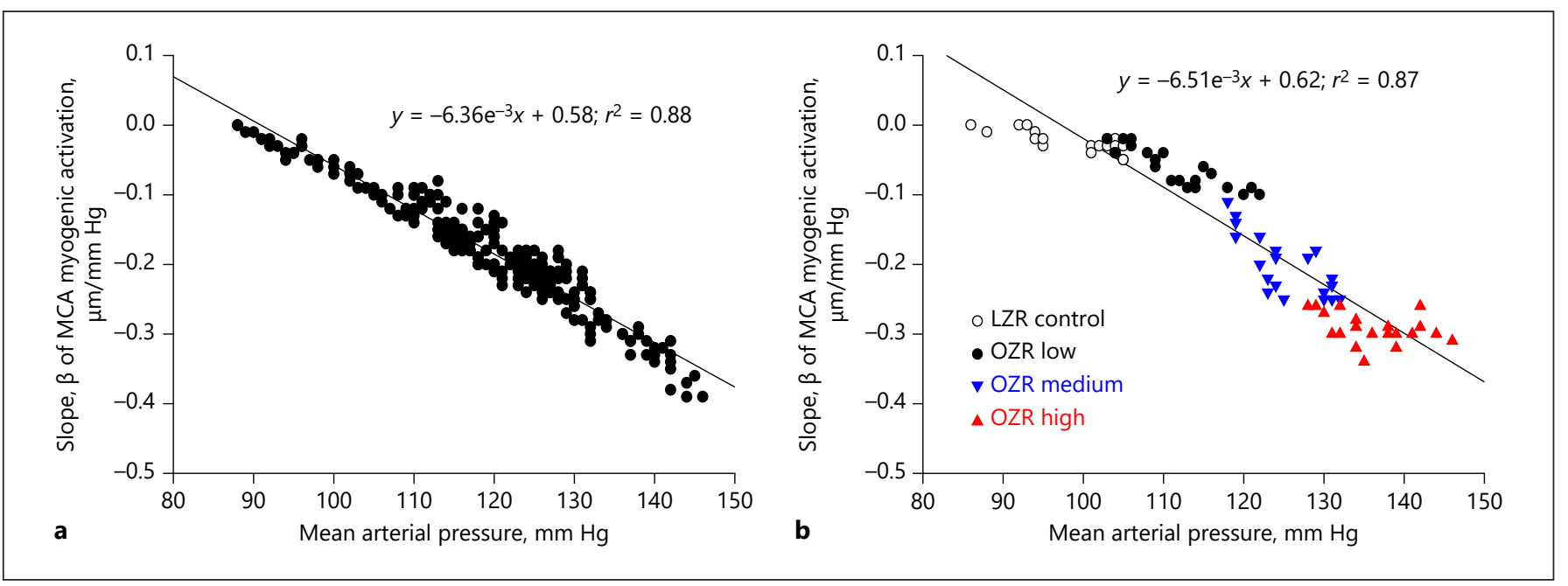

Fig. 1. Scatter plots, with regression lines of best fit through the data, describing the correlation between mean arterial pressure and the slope ( $\beta$ ) of MA in MCA of OZR. a Historical data in OZR. $\mathbf{b}$ The relationship from each animal used in the present study (see the text for details). OZR, obese Zucker rat; MA, myogenic activation; MCA, middle cerebral artery; LZR, lean Zucker rat.

Table 1. Baseline characteristics of animals used in the present study

\begin{tabular}{|c|c|c|c|c|c|}
\hline & $\begin{array}{l}\text { LZRs } \\
(n=20)\end{array}$ & $\begin{array}{l}\text { OZRs } \\
(n=60)\end{array}$ & $\begin{array}{l}\text { OZRs } \\
(n=20) \text {, "low" }\end{array}$ & $\begin{array}{l}\text { OZRs } \\
(n=20), \text { "medium" }\end{array}$ & $\begin{array}{l}\text { OZRs } \\
(n=20), \text { "high" }\end{array}$ \\
\hline Mass, g & $366 \pm 11$ & $712 \pm 23^{*}$ & $707 \pm 20^{*}$ & $716 \pm 24^{*}$ & $711 \pm 21^{*}$ \\
\hline$(\text { Glucose })_{\text {blood }}, \mathrm{mg} / \mathrm{dL}$ & $86 \pm 7$ & $164 \pm 18^{*}$ & $164 \pm 16^{*}$ & $159 \pm 20^{*}$ & $167 \pm 17^{*}$ \\
\hline$(\text { Insulin) })_{\text {plasma }}, \mathrm{ng} / \mathrm{mL}$ & $0.4 \pm 0.1$ & $7.91 \pm 1.1^{*}$ & $7.6 \pm 0.9^{*}$ & $8.1 \pm 1.1^{*}$ & $7.9 \pm 1.2^{*}$ \\
\hline Nitrotyrosine, pg/mL & $22.3 \pm 3.5$ & $47.7 \pm 5.8^{*}$ & $46.8 \pm 6.2^{*}$ & $49.4 \pm 5.9^{*}$ & $50.6 \pm 6.1^{*}$ \\
\hline
\end{tabular}

OZR, obese Zucker rat; LZR, lean Zucker rat. * $p<0.05$ versus LZR. ${ }^{\dagger} p<0.05$ versus OZR “low."

Mechanical responses following challenge with logarithmically increasing dosages of acetylcholine or adenosine were fit with the three-parameter logistic equation

$$
y=\min +\left[\frac{\max -\min }{1+10^{\log E D_{50}-x}}\right],
$$

where y represents the vessel diameter; "min" and "max" represent the lower (minimum) and upper (maximum) bounds, respectively, of the change in diameter with agonist concentration; $x$ is the logarithm of the agonist concentration; and $\log \mathrm{ED}_{50}$ represents the logarithm of the agonist concentration $(x)$ where the response $(y)$ is halfway between the bounds. For the presentation of results, we have focused on the changes in the upper bounds as a representation of vessel reactivity as lower bound remained consistent between all groups (defined as the pre-challenge diameter), and we did not determine a consistent or significant change to the $\log \mathrm{ED}_{50}$ values between treatment groups. Using this approach, the upper bound rep-

Metabolic Syndrome and Cerebral Vasculopathy resents the statistically determined asymptote for the concentration response relationship and does not assume that the vascular response at the highest utilized concentration of the agonist represents the maximum possible response. Rather, the sigmoidal relationship of best fit to the data will predict the statistical upper bound of the response given the data points entered into the model. As such, the upper bound is often slightly larger than the dilator response of the vessel at the highest concentration of the agonist.

The myogenic properties of MCA from each experimental group were plotted as mean diameter at each intraluminal pressure and fitted with a linear regression $\left(y=\alpha_{0}+\beta x\right)$, where the slope coefficient $\beta$ represents the degree of MA ( $\Delta$ diameter/ $\Delta$ pressure). Increasingly negative values of $\beta$, therefore, represent a greater degree of MA in response to changes in intralumenal pressure. A similar analysis was used to determine $\mathrm{NO}$ and $\mathrm{H}_{2} \mathrm{O}_{2}$ bioavailability in response to increasing concentrations of methacholine, where $\beta$ represents the rate of change of the metabolite released by the vessels in response to agonist challenge. 


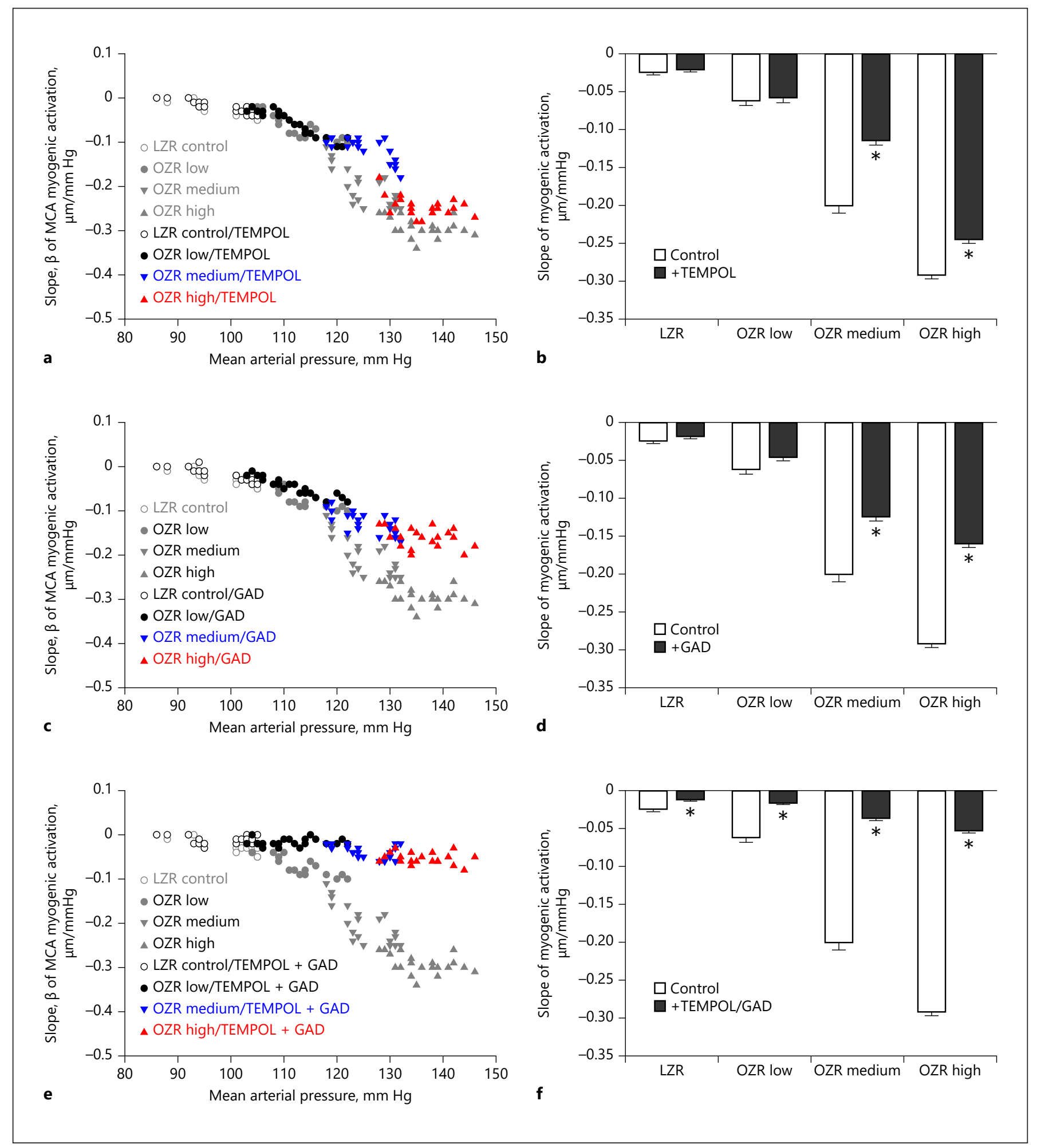

Fig. 2. Data describing the relationship between mean arterial pressure and the slope $(\beta)$ of MA in MCA of OZR. Untreated control data (from Fig. 1, b) are grayed out, and the impact on $\beta$ from individual OZR is presented in response to pretreatment with TEMPOL (a), gadolinium (c), and both treatments combined (e).

b, $\mathbf{d}, \mathbf{f}$ The aggregate data within the different groups for those treatments, respectively. Data are presented as mean $\pm \mathrm{SE}^{*} p<0.05$ versus control responses. $n=20$ vessels/group. OZR, obese Zucker rat; MA, myogenic activation; MCA, middle cerebral artery; LZR, lean Zucker rat. 


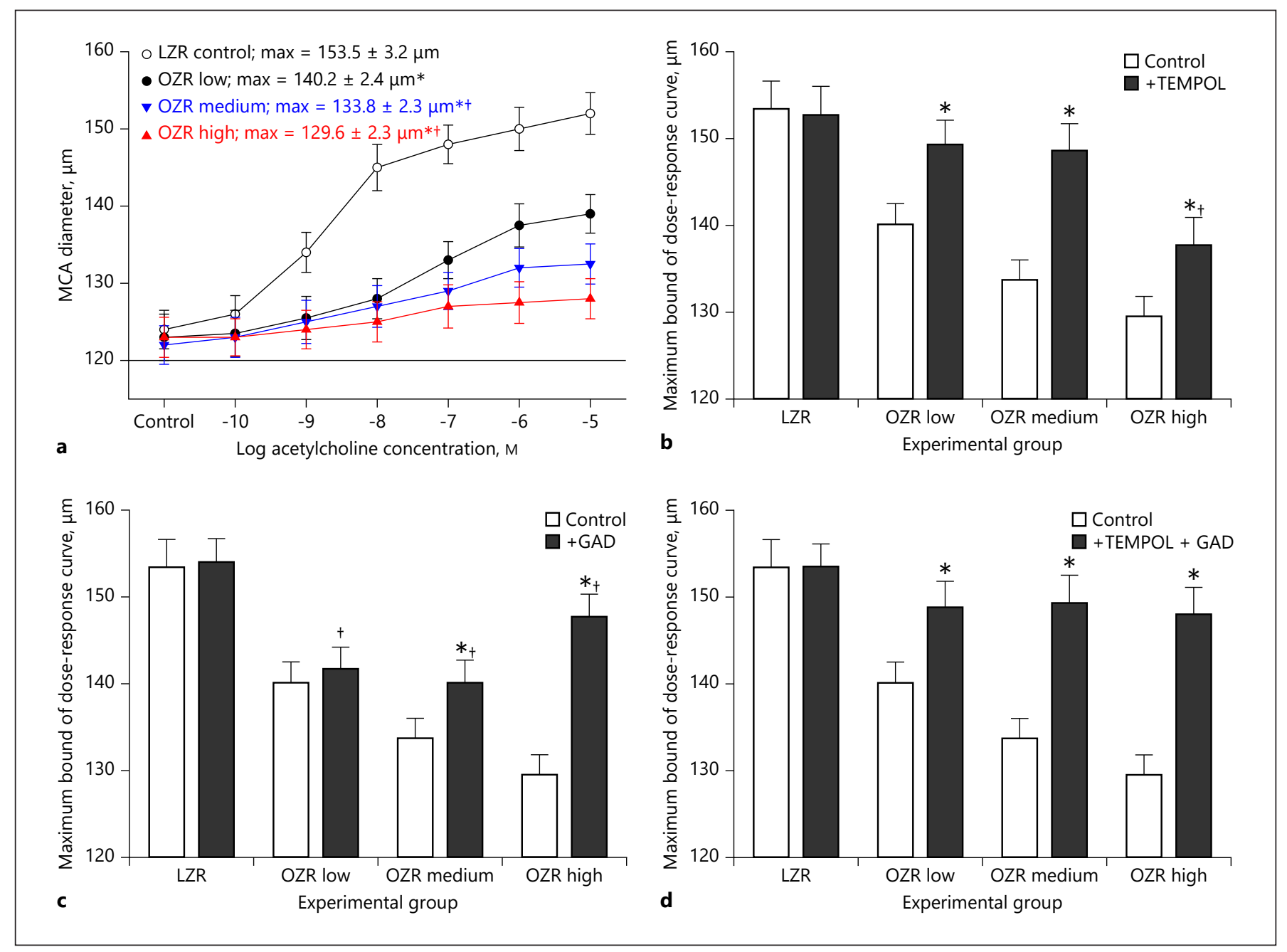

Fig. 3. Dilator responses of ex vivo MCA from LZR and OZR in response to increasing concentrations of acetylcholine. a Changes in diameter of MCA from LZR and the 3 groups of OZR following challenge with acetylcholine. $\mathbf{b}$-d Changes in the upper bound of the acetylcholine response in all groups following pretreatment of

To assess the relationship of wall tension with intravascular pressure, vessel wall thickness was calculated as

$$
\mathrm{WT}=\frac{(\mathrm{OD}-\mathrm{ID})}{2},
$$

where WT represents wall thickness $(\mu \mathrm{m})$ and OD and ID represent the outer and inner diameter $(\mu \mathrm{m})$ of the artery, respectively.

Vascular wall tension $(T ; \mathrm{dyn} / \mathrm{cm})$ was calculated as

$$
T=\left(P_{\mathrm{IL}} \times \frac{\mathrm{ID}}{2}\right) / \mathrm{WT},
$$

where pressure is determined in dynes $/ \mathrm{cm}^{2}(1 \mathrm{~mm} \mathrm{Hg}=1,333.2$ $\left.\mathrm{dyn} / \mathrm{cm}^{2}\right)$, and all measurements for ID and WT follow the procedures outlined above. The slope of vascular wall tension versus

Metabolic Syndrome and Cerebral Vasculopathy
MCA with TEMPOL, gadolinium, or both agents combined, respectively. Data are presented as mean $\pm \mathrm{SE} * p<0.05$ versus control responses in that group; $\dagger p<0.05$ versus LZR. $n=16-20$ vessels/group. OZR, obese Zucker rat; MCA, middle cerebral artery; LZR, lean Zucker rat.

intraluminal pressure $\left(P_{\mathrm{IL}}\right)$ was determined using the linear regression analyses outlined above.

All data are presented as mean \pm SE. Differences in all slope coefficients or descriptive characteristics between the LZR and OZR groups were assessed using ANOVA, with the Student-Newman-Keuls post hoc test, or individual $t$-tests, as appropriate. In all cases, $p<0.05$ was taken to reflect statistical significance.

\section{Results}

Table 1 summarizes the baseline characteristics of animals used in the present study. At $\sim 17$ weeks of age, OZRs were significantly heavier than their age-matched 

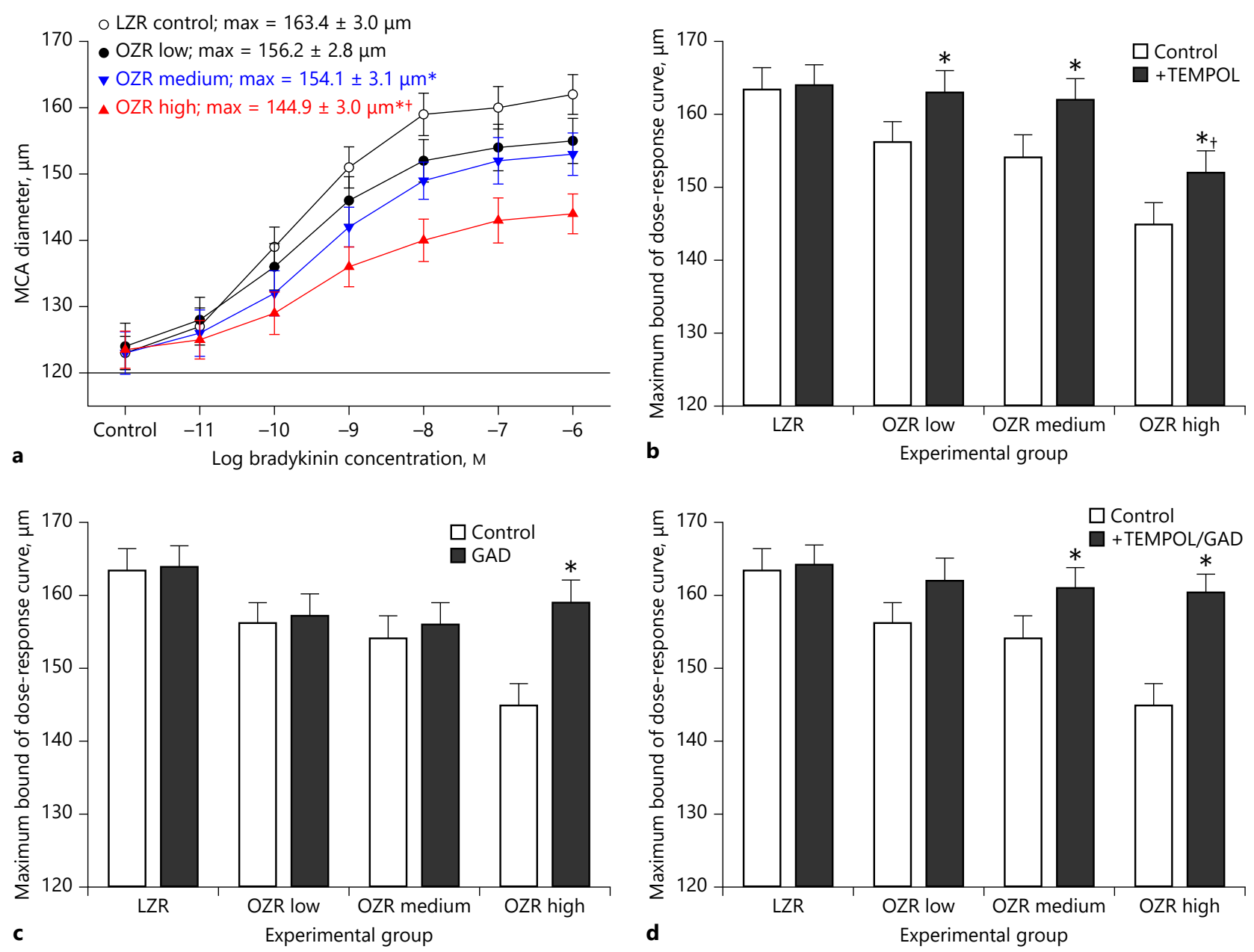

Fig. 4. Dilator responses of ex vivo MCA from LZR and OZR in response to increasing concentrations of bradykinin. a Changes in diameter of MCA from LZR and the 3 groups of OZR following challenge with bradykinin. $\mathbf{b}$-d Changes in the upper bound of the bradykinin response in all groups following pretreatment of MCA

LZRs. In addition, OZRs exhibited many of the characteristics of the metabolic syndrome, including impaired glycemic control, moderate hypertension, and a significant pro-oxidant and pro-inflammatory condition present in the blood.

Figure 2 summarizes MA of ex vivo MCA from the OZR and LZR in the present study under control conditions and following pretreatment of vessels with TEMPOL, gadolinium, or both agents. Following acute treatment with TEMPOL, there was a modest reduction to the severity of MA in the medium and high groups for OZR $\mathrm{MCA}$, suggesting a contributing role for oxidant stress in

with TEMPOL, gadolinium, or both agents combined, respectively. Data are presented as mean $\pm \mathrm{SE}^{*} p<0.05$ versus control responses in that group; $\dagger p<0.05$ versus LZR. $n=16-19$ vessels/ group. OZR, obese Zucker rat; MCA, middle cerebral artery; LZR, lean Zucker rat.

altering MA, with no impact in the low group (Fig. 2a, b). Application of gadolinium to the ex vivo MCA of OZR significantly reduced the sensitivity of MA in both the medium and high groups (Fig. 2c, d). Combined application of TEMPOL and gadolinium reduced the slope of MA of MCA from all groups of OZR to a point that was not different from that in untreated LZR (Fig. 2e, f).

Dilator responses of ex vivo MCA in response to increasing concentrations of acetylcholine were impaired in all OZR groups compared to those in LZR, with the high OZR group having the most pronounced impairment (Fig. 3a). After acute treatment of TEMPOL, dilator 


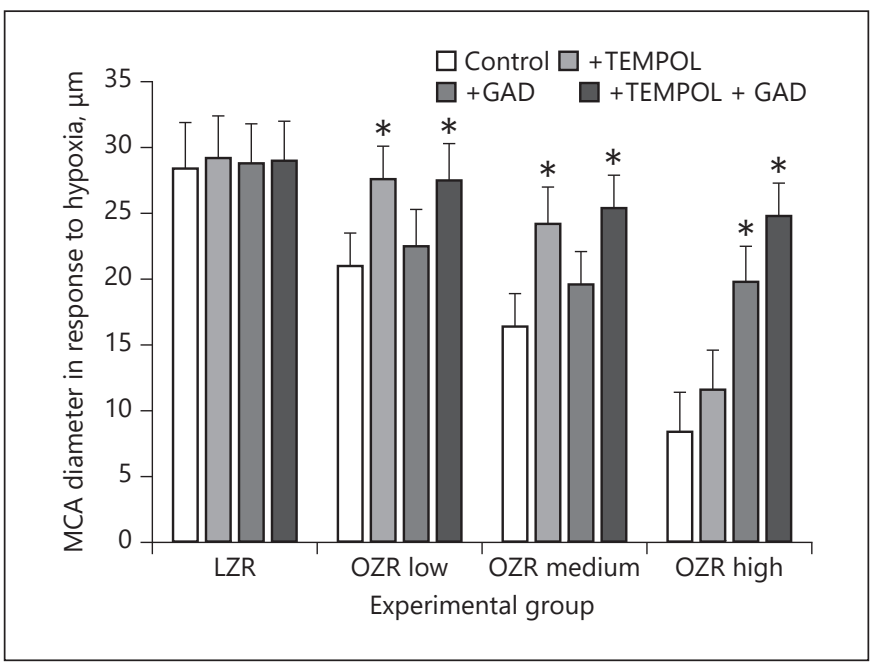

Fig. 5. Dilator responses of ex vivo MCA from LZR and the 3 groups of OZR in response to decreased $\mathrm{PO}_{2}$. Also presented in this figure are the responses to hypoxia following pretreatment of MCA with TEMPOL, gadolinium, or both agents combined. Data are presented as mean $\pm \mathrm{SE}^{*} p<0.05$ versus control responses in that group. $n=15-20$ vessels/group. OZR, obese Zucker rat; MCA, middle cerebral artery; LZR, lean Zucker rat.

responses of MCA from the OZR groups were improved compared to their untreated control responses, and the degree of improvement of acetylcholine-induced dilation in the OZR low and medium groups was not significantly different from that in untreated LZRs (Fig. 3b).

The impact of gadolinium treatment was quite different from that for TEMPOL. Gadolinium treatment had a minimal impact on dilator responses of vessels in either the low or medium MA groups but caused a very large improvement in dilator reactivity in those OZR MCAs exhibiting a high MA under untreated conditions (Fig. 3c). Combined treatment of vessels with both TEMPOL and gadolinium resulted in acetylcholine-induced responses that were comparable to those in LZR controls (Fig. 3d).

Figure 4 summarizes the dilation of isolated MCA in response to increasing concentrations of bradykinin. While responses to bradykinin were generally more robust than those for acetylcholine, a comparable pattern of impaired responses to this endothelium-dependent agonist was identified (Fig. 4a). TEMPOL treatment largely normalized vascular responses to bradykinin in both the low and medium groups of OZR but produced only a modest (but statistically significant) improvement in the high group (Fig. 4b). In contrast, treatment of the MCA with gadolinium had minimal impact on bradykinin-induced dilation in the low and medium groups, but significantly improved responses in the high MA in OZR MCA (Fig. 4c). Combined application of TEMPOL and gadolinium improved to bradykinin-induced dilation to a comparable degree in all groups (Fig. 4d).

While dilator responses to hypoxia of MCA from OZR were consistently reduced from those in LZR, the degree of this reduction was correlated with the sensitivity of MA (Fig. 5). Acute treatment of the isolated MCA with TEMPOL caused a significant improvement of hypoxic dilation in MCA from OZR, manifesting a low or medium elevation in MA but had no impact on vessels that had a high MA. A reversal of this pattern was evident following treatment with gadolinium, where the improvements of hypoxic dilation were modest in MCA of OZR with low or medium elevations in MA and significantly improved in vessels with high MA. Combined treatment of OZR MCA with gadolinium and TEMPOL resulted in an improved response to hypoxia regardless of the initial severity of MA.

Figure 6 summarizes the data describing the impact of altered MA in MCA of OZR on adenosine-induced dilation. Under untreated conditions, increasing concentrations of adenosine resulted in a normal dilator response with the exception of a reduced response in MCA from OZR with the highest levels of MA (Fig. 6a). Pretreatment of the MCA with TEMPOL (Fig. 6b), gadolinium (Fig. 6c), or the combination of both (Fig. 6d) had no significant impact on the dilator response to adenosine compared to untreated control conditions.

In conduit arteries, treatment with increasing concentrations of methacholine resulted in significantly less NO release and an elevated release of $\mathrm{H}_{2} \mathrm{O}_{2}$ in OZR compared to responses in arteries from LZR (Fig. 7). The magnitude of these shifts in $\mathrm{NO}$ and $\mathrm{H}_{2} \mathrm{O}_{2}$ release was reduced in vessels from the OZR high group. In pooled cerebral and conduit arteries from OZR following treatment with arachidonic acid, the production and release of PGI2 (from 6-keto-PGF $1_{\alpha}$ ) was attenuated in all groups of arteries from OZR although least in those from the high MA group. In contrast, the elevation in TxA2 production (from 11-dehydro-TxB2), although significant in all groups, was increased to the least extent in vessels from OZR in the high MA group.

The changes in calculated vascular wall tension in MCA from LZR and OZR following increased intralumenal pressure in the present study are summarized in Figure 8 . The slope of the wall tension versus intravascular pressure relation was highest in MCA from LZR and ex- 

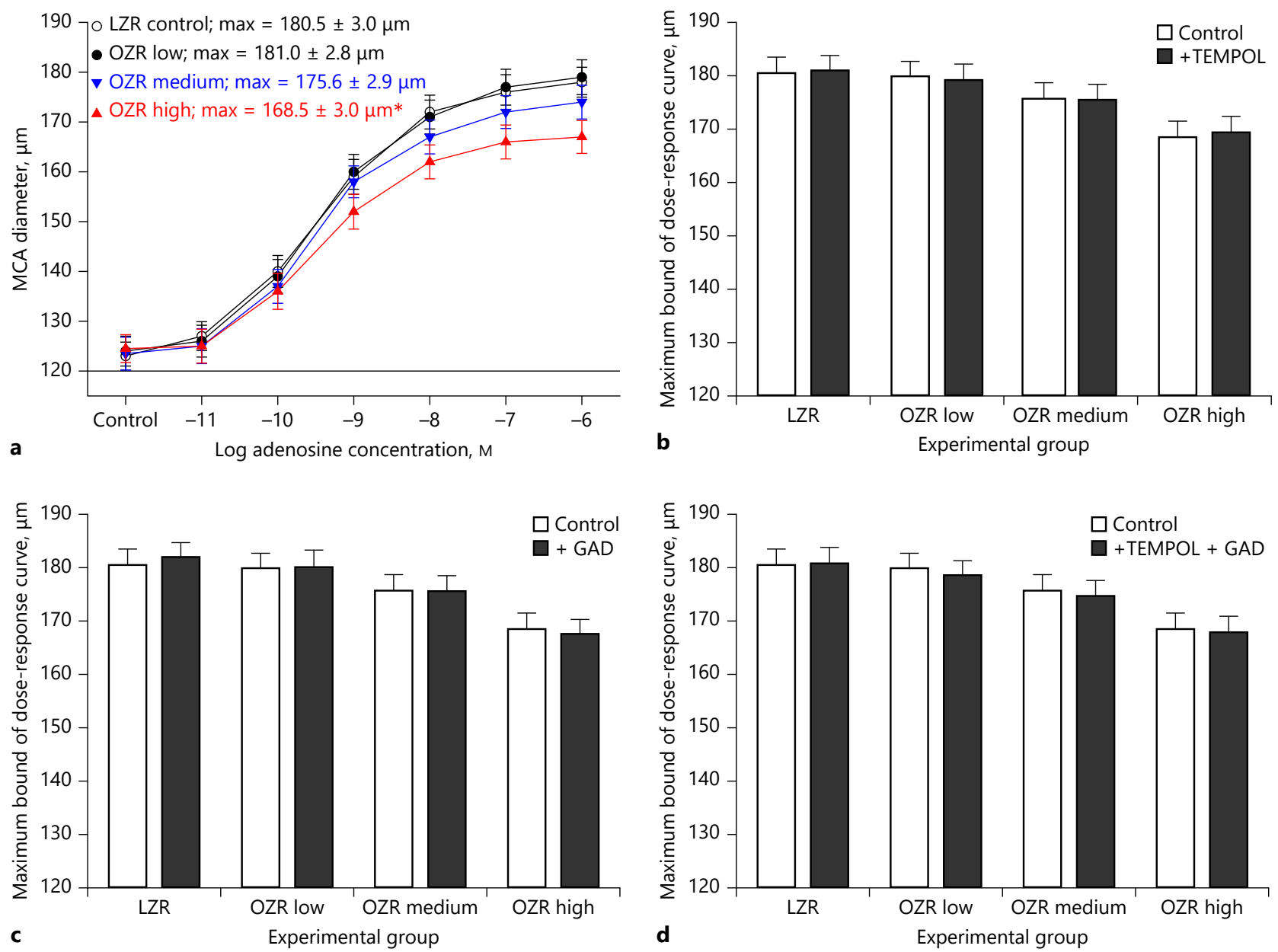

Fig. 6. Dilator responses of ex vivo MCA from LZR and OZR in response to increasing concentrations of adenosine. a Changes in diameter of MCA from LZR and the 3 groups of OZR following challenge with adenosine. $\mathbf{b}-\mathbf{d}$ Changes in the upper bound of the adenosine response in all groups following pretreatment of MCA

hibited a gradual reduction in slope across the groups of OZR to the point where this relationship became statistically significant in the OZR high group. This suggests that the proportionate increase in the severity of MA in MCA of OZR is greater than the elevation in arterial pressure at this age.

Figure 9 summarizes the predictive ability of plasma insulin concentration (Fig. 9a), TNF- $\alpha$ (Fig. 9b), and nitrotyrosine (Fig. 9c) on the sensitivity of MA ( $\beta$ ) across all animals in the present study. As presented in the 3 panels, none of these variables associated with glycemic control, chronic inflammation, or vascular oxidant stress load were well correlated with slope of MA.

with TEMPOL, gadolinium, or both agents combined, respectively. Data are presented as mean \pm SE $n=16-18$ vessels/group. OZR, obese Zucker rat; MCA, middle cerebral artery; LZR, lean Zucker rat.

The relationship between the severity of MA $(\beta)$ and endothelial function, estimated as the upper bound of the acetylcholine concentration-response curve, and the magnitude of hypoxic dilation are presented in Figure 10. While both estimates of endothelial function were significantly reduced in all groups of OZR compared to the responses in LZR, there was a clear change in the slope of this relationship, such that the greatest MA was associated with the greatest reduction in vasodilator responses to acetylcholine or reduced $\mathrm{PO}_{2}$. 


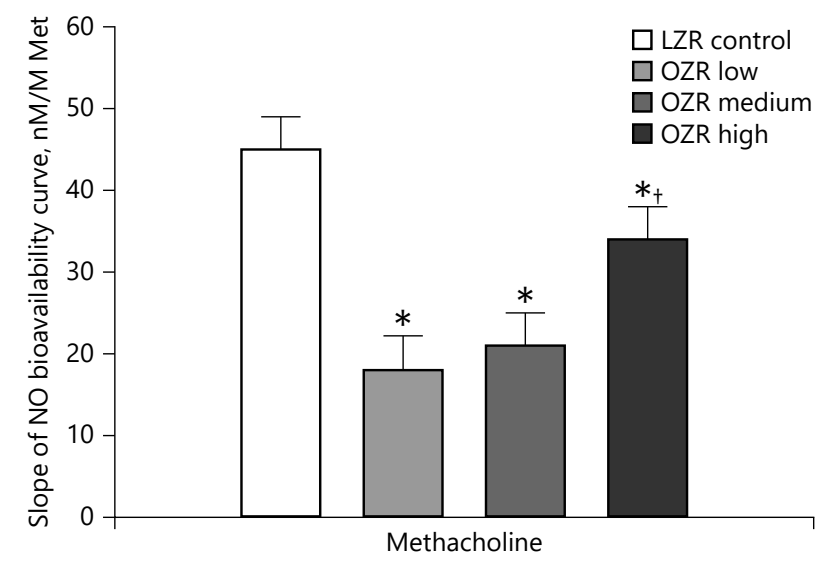

a

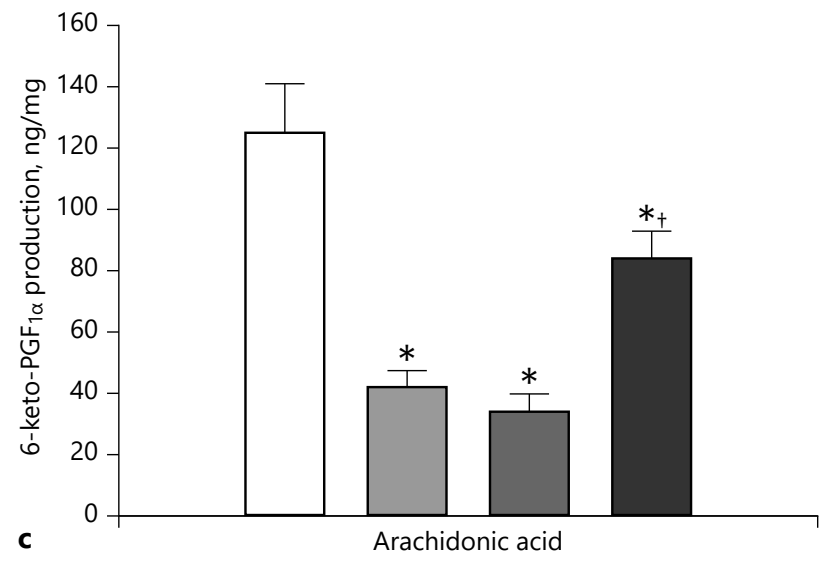

Fig. 7. Data describing the bioavailability of vasoactive metabolites using ex vivo cerebral and conduit arteries from LZR and OZR used in the present study. The arterial productions of $\mathrm{NO}(\mathbf{a})$ and hydrogen peroxide $\left(\mathrm{H}_{2} \mathrm{O}_{2} ; \mathbf{b}\right)$ are presented as the slope of the relationship in response to increasing concentrations of methacho-

\section{Discussion}

The primary findings of the present study begin with the observation that the dilator responses of the MCA to endothelium-dependent stimuli were attenuated in OZR, with the initial interpretation that the severity of the attenuation was roughly correlated with the extent to which MA was increased from normal. As presented in Table 1, while the other measured constituents of the metabolic syndrome were increased in OZR compared to those in LZR, they did not exhibit a demonstrable heterogeneity as was the case for arterial pressure at $\sim 17$ weeks of age. This suggest that, at this age and severity of the metabolic syndrome, our approach of using the variability in MA

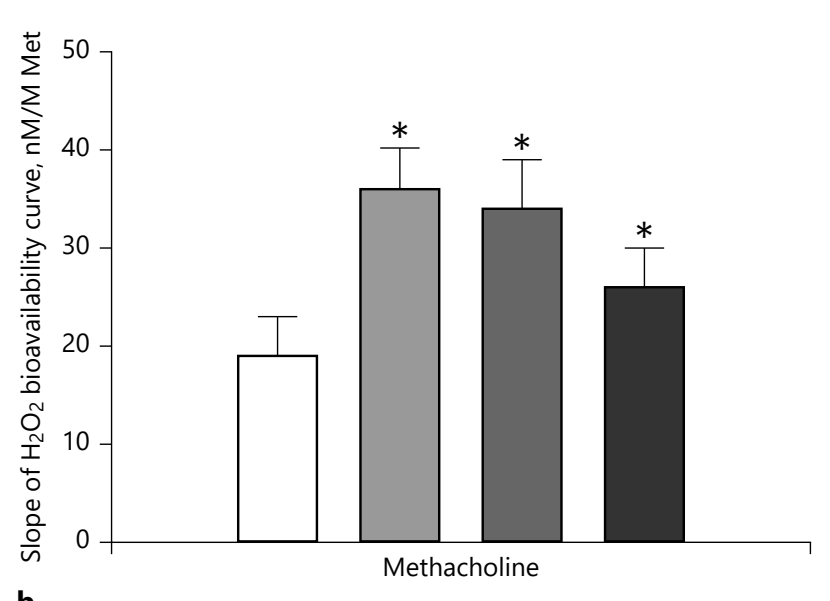

b

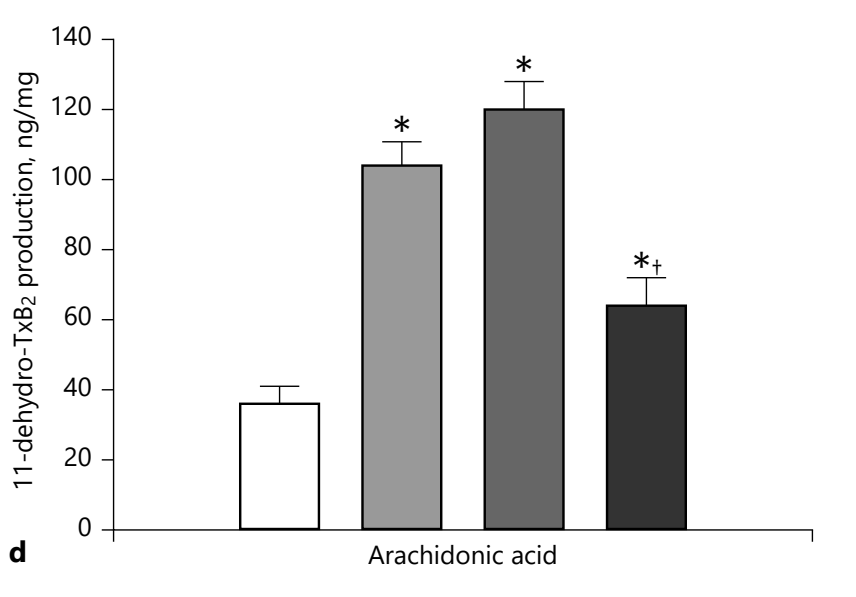

line. c, $\mathbf{d}$ Arterial production of $\mathrm{PGI}_{2}$ (as 6-keto- $\mathrm{PGF}_{1 \alpha}$ ) and $\mathrm{TxA}_{2}$ (as 11-dehydro $\mathrm{TxB}_{2}$ ) following challenge with arachidonic acid. Data are presented as mean $\pm \mathrm{SE} * p<0.05$ versus LZR; $\uparrow p<0.05$ versus OZR low. $n=15-18$ vessels/group (see the text for details). OZR, obese Zucker rat; LZR, lean Zucker rat; NO, nitric oxide.

(as opposed to other parameters) as the framework for analyses and to gain further understanding into how integrated vascular tone emerges is a reasonable one. Treatment of MCA from OZR with the anti-oxidant TEMPOL significantly improved responses to endothelium-dependent stimuli in groups where the increase in MA was "low" or "medium," but had a more modest effect in vessels with the "high" level of MA. In contrast, treatment of MCA with the mechanosensitive cation channel inhibitor gadolinium chloride to reduce the enhanced MA in MCA of OZR to levels comparable to that in control LZR had a modest impact on endothelial dependent dilation in MCA from the "low" and "medium" MA groups, but improved endothelium-dependent responses in MCA from 


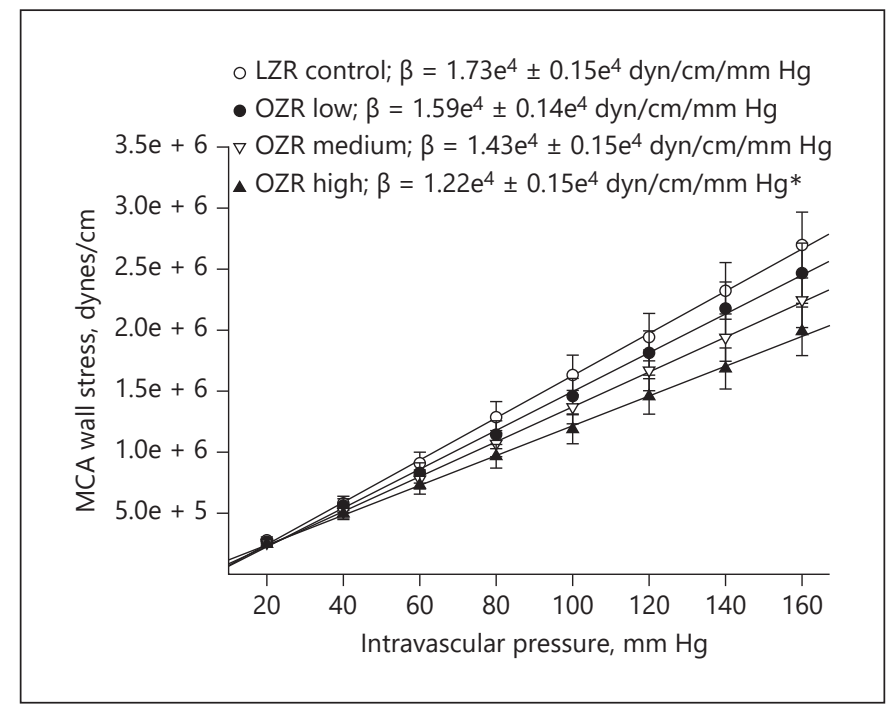

Fig. 8. Data describing the relationship between intravascular pressure and wall stress experienced by MCA for LZR and the 3 groups of OZR in the present study. The regression line of best fit is presented for each group and data are presented as mean \pm SE. The slopes $(\beta)$ of the regression lines are presented in the figure legend. ${ }^{*} p<0.05$ versus LZR. $n=20$ vessels/group. OZR, obese Zucker rat; MCA, middle cerebral artery; LZR, lean Zucker rat.

the "high" group. Combined treatment of OZR MCA with both gadolinium chloride and TEMPOL resulted in endothelium-dependent responses that were very comparable across groups and with those from control LZR. While treatment with TEMPOL and gadolinium has previously been shown to improve dilator responses and reduce MA respectively $[8,23]$, our efforts at manipulating the severity of MA in the acute setting may allow for greater insight into the nature of vascular behavior under normal and pathophysiological conditions.

Metabolic syndrome is a chronic disease that can lead to long-term elevations in cardiovascular and cerebrovascular disease that may manifest themselves in the form of stroke, transient ischemic attack, or inappropriate cerebral perfusion. Precise control of cerebral vascular tone that involves the integration of various signals from the endothelium, vascular smooth muscle, and other sources to determine an appropriate level of tone is a crucial contributor to these poor outcomes. The MCA is a vessel of particular interest because of its significant role in cerebral blood flow regulation and because it is a frequent site of occlusion/stroke [23]. As such, altered patterns of reactivity of MCA with metabolic syndrome provide insight into how altered aspects of disease development can impair blood flow to the brain or impair perfusion within the brain. As a complement to these data, measurements of vascular metabolites were taken from pooled conduit arteries from LZR and OZR as correlative evidence of altered endothelial function in order to gain insight into mechanistic pathways where the signal to be determined may be difficult to distinguish in an isolated resistance vessel.

A fundamental observation from the present study is that the severity of MA of the MCA from OZR manifesting the metabolic syndrome is well-predicted by the elevation in blood pressure (hypertension) within that animal. This is a well-established concept within resistance arterioles in multiple models that can most easily be seen as a protective mechanism to control the extent to which pressure waves penetrate into the microvascular networks $[8,10]$. Our division of the increase in MA into thirds ("low," "medium," and "high") was intended to provide a greater ability to integrate MA and endothelial function in metabolic syndrome by reducing variance around the mean responses within groups. The subsequent use of TEMPOL and gadolinium was selected to evaluate well-established mechanisms for improving endothelial function and blunting MA, respectively, in OZR. With this approach, it was evident that "low" and "medium" increases in MA, while having the ability to impact dilator reactivity in MCA of OZR, had less significance than the increased pro-oxidant and pro-inflammatory environment that accompanies metabolic syndrome. However, in the "high" MA group, the beneficial impact of the antioxidant treatment (TEMPOL) was unexpectedly less effective (even after gadolinium treatment) to normalize myogenic influences to the levels determined in control LZR.

In terms of the impact on dilator reactivity, MCA from OZR exhibited a reduced reactivity to increasing concentrations of acetylcholine and bradykinin and to reduced oxygen tension, but also demonstrated a largely intact response to increasing concentrations of adenosine (an endothelium-independent response). However, contrary to what has been commonly assumed, the beneficial impact of anti-oxidant treatment (TEMPOL) on dilator responses of MCA from OZR was greatest in the "low" and "medium" groups for increased MA, with a comparatively smaller impact in the "high" group, even when the increased MA was removed (gadolinium). With the severity of MA broken into thirds, this was a particularly striking observation that seems to suggest that dilator responses in this group are more constrained by myogenic tone than by the impairment of endothelial function by oxidative stress. It should be mentioned that the dose of 


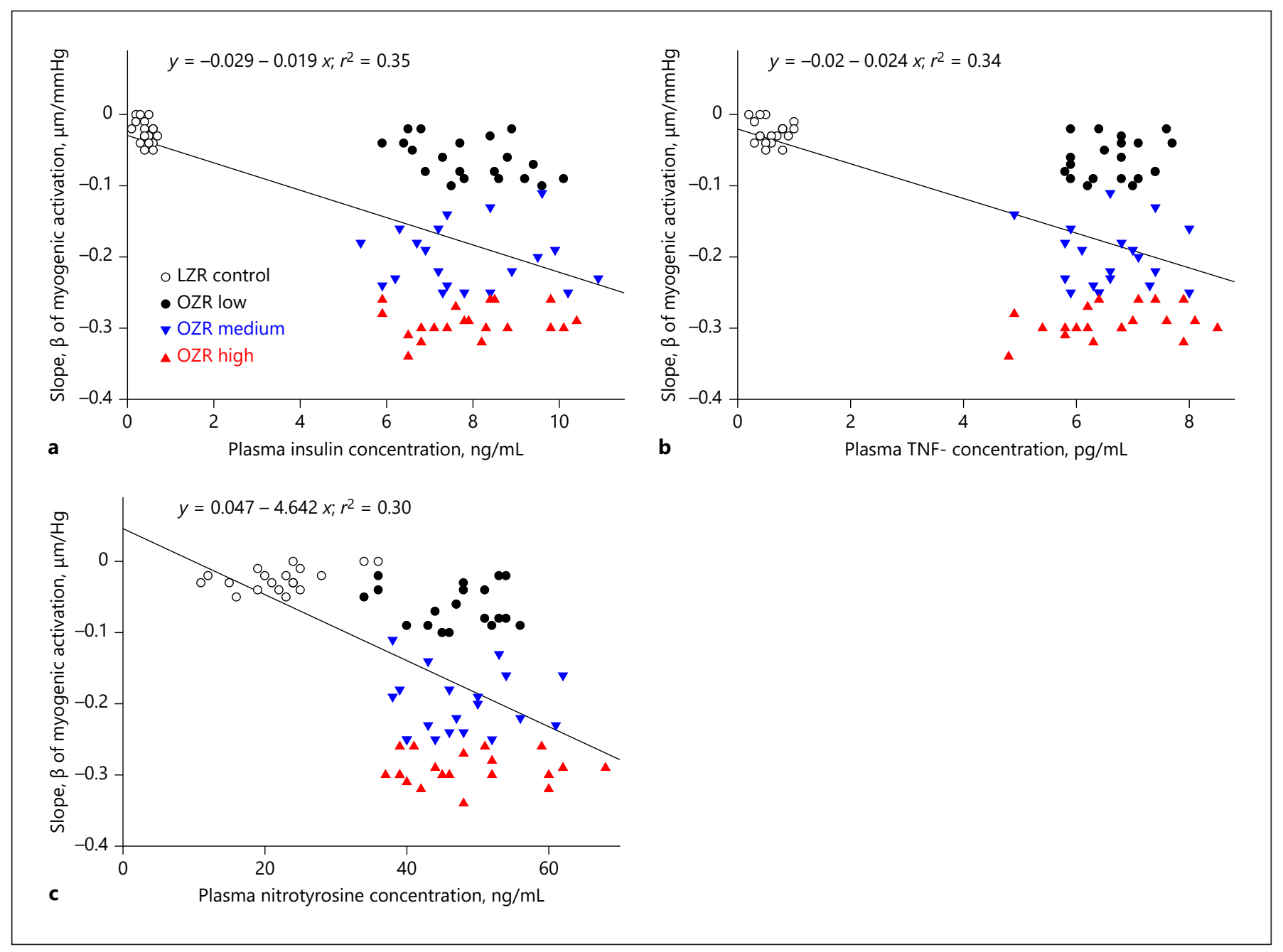

Fig. 9. Scatter plots presenting the correlation between plasma insulin concentration (a), TNF- $\alpha$ concentration (b), or nitrotyrosine concentration (c) and the slope ( $\beta$ ) of MA of LZR and OZR in the present study. The regression line of best fit for each relation is presented within each panel. OZR, obese Zucker rat; MA, myogenic activation; LZR, lean Zucker rat.

gadolinium used for an individual vessel was not constant and was titrated to result in a change in myogenic sensitivity. As such, the present study does not allow us to differentiate the impact of gadolinium on endothelial function independent of MA. In addition, gadolinium also has the ability to inhibit L-type Ca2+ and TRP channels in addition to stretch-activated cation channels, so this should be kept in mind when interpreting the results from this study.

While direct relevance of the above observation to mechanistic interpretation is imperfect, some insight may be gained through the measurements of vasoactive metabolites in larger cerebral and conduit arteries. As shown in Figure 7, endothelial production of NO, a major metabolite responsible for the dilator responses to acetylcholine and bradykinin, is reduced in all groups of OZR, but is maintained at a higher level in OZR with stronger levels of MA. Interestingly, a roughly inverse relationship was determined with regard to $\mathrm{H}_{2} \mathrm{O}_{2}$ production, with measured levels being higher in the "low" and "medium" groups with a somewhat reduced production in the group with "high" MA. Given that $\mathrm{H}_{2} \mathrm{O}_{2}$ is frequently proposed to be a compensatory or "backup" dilator signaling metabolite under conditions of compromised NO bioavailability $[24,25]$, these observations may suggest that the magnitude of endothelial dysfunction in the "high" group may not be as severe as predicted (supporting the presented interpretation of the data from the TEMPOL treat- 

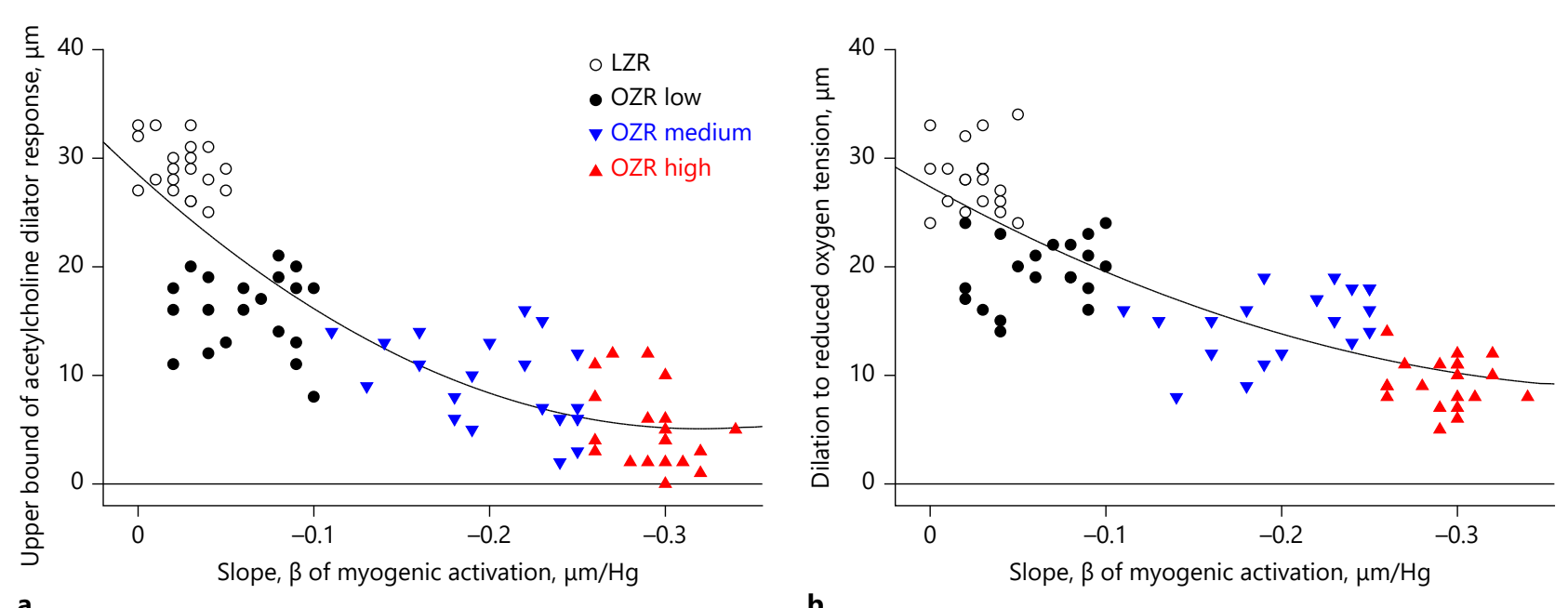

a

b

Fig. 10. Scatter plots presenting the correlation between the slope $(\beta)$ of MA of LZR and OZR and endothelial function. Endothelial function was estimated as the upper bound of the acetylcholine response curve (a) or the dilator response to hypoxia (b) for ex vivo MCA. The 2nd order regression line of best fit for each relation is presented within each panel. OZR, obese Zucker rat; MA, myogenic activation; MCA, middle cerebral artery; LZR, lean Zucker rat.

ments). A comparable interpretation may be appropriate regarding the data describing altered arachidonic acid metabolism. As previous studies have clearly indicated that arachidonic acid metabolism to PGI2 is a major contributor to hypoxic dilation in MCA16, we and others have determined that the increased production of TxA2 in combination with a reduced production of PGI2, owing to the pro-inflammatory and pro-oxidant environment of the metabolic syndrome in OZR is a major contributor to the impaired vascular responses to hypoxia [21]. While these previous interpretations of results are supported by the present data, here our results suggest a more subtle relationship wherein the increased MA of larger resistance arterioles is associated with less severe dysfunction of the vascular endothelium as a result of the environment within metabolic syndrome. It is important to emphasize that these vascular metabolite data were not taken from the MCA of the OZR under study, but rather from other cerebral and conduit arteries. As such, these results should be interpreted conservatively.

These results lead to general questions of whether increases in the sensitivity of MA $(\beta)$ of MCA of OZR confer a degree of protection from endothelial dysfunction in the face of the endocrine, inflammatory and oxidant derangements - and how this would be manifest. One possibility, presented in Figure 8, is that vascular wall tension in MCA is maintained at a progressively lower level with increasing mean arterial pressure in OZR. The initial interpretation of these data is that the rate at which the sensitivity of MA increases exceeds the rate increasing mean arterial pressure and thus reduces vascular wall tension in the MCA with hypertension (reflected in the reduced slope of the relationship over the ranges of intralumenal pressure). What appears to be remarkable is that the unexpected maintenance of endothelial function associated with this reduction in wall tension occurs despite the fact that levels of impaired glycemic control, inflammation, and oxidant stress are extremely comparable across the different groups of MA in OZR. Speculating, it may be that the increased MA, especially in the more severe cases of hypertension in OZR, confers a protection to vascular reactivity (and the downstream microvascular networks) in the face of an extremely challenging environment for endothelial function. This compensatory response may act to prevent a complete collapse of vascular function and to maintain integrated microvascular function to the extent that this is possible. The increases in MA in the "high" group may be an attempt to maintain vascular function to the extent possible by optimizing wall stress and tension, and attempting to keep these variables within an ideal range for maintaining overall vascular reactivity - a concept originally introduced by Gore [26]. This 
speculation may be further supported by the data presented in Figure 10, where the steepest decline in endothelial function tends to occur between control LZR and OZR with "low" changes in MA, with the remainder of the relation being much flatter, despite the increased myogenic responses in "medium" and "high" groups.

Although outside the scope of this study, it is important to recognize that our focus on endothelial function as a means for determining vascular diameter is likely somewhat limited in overall perspective. Endothelial function (and especially its interaction with MA) is critical for the determination of vascular diameter, resistance to blood flow, and perfusion distribution within an organ/tissue. However, the importance of endothelial function for coordinating vascular signaling [27], maintaining barrier integrity and permeability [28], modulating thrombosis and platelet activation, and determining microvascular network growth/regression [29] cannot be ignored. These other issues, merely given as a few examples, all take on a magnified importance under challenged pathological conditions and for human health outcomes viewed from a more translational perspective. As such, gaining insight into how specific changes in endothelial function may impact these roles of the endothelium will be a critical area for future investigation.

In summary, the results of the present study clearly demonstrate an increased MA of MCA from OZR that is correlated with the severity of the hypertensive component of the metabolic syndrome that develops in this model. While endothelium-dependent dilator reactivity of MCA from OZR to multiple stimuli is significantly attenuated, the contributions of the pro-oxidant/pro-inflammatory environment and an increased myogenic tone are variable between animals. Under conditions where MA is the most severe, endothelial function appears to receive some degree of protection despite the challenging environment, which may reflect the ability of the MCA to reduce wall tension despite the increased intravascular pressure. The impact that the protective effect on the vascular endothelium of OZR with the greater degrees of hypertension has on other aspects of vascular/ microvascular function will require further investigation. The results of the present study also highlight the importance of considering data from both an aggregate perspective and within the individual animal, to the extent possible, especially when considering the impact of elevated disease risk conditions.

\section{Statement of Ethics}

All procedures in this manuscript were evaluated by the responsible institutional review committees. The research presented was conducted ethically in accordance with the World Medical Association Declaration of Helsinki and following the Animal Research Reporting of in vivo Experiments (ARRIVE) guidelines and the appropriate review by institutional review bodies.

\section{Conflict of Interest Statement}

The authors declare no conflict of interest.

\section{Funding Sources}

This work was supported by NIH R01-HL128242-01 (JHL); NIH, BINP R56 NS117754-01 and NIHGMS 5U54GM104942-03 (PDC); and CIHR \#389769, NSERC RGPIN-2018-05450, NIH R01-DK64668, and AHA EIA 0740129N (JCF).

\section{Author Contributions}

Each author has (1) made substantial contributions to the conception or design of the work or to the acquisition, analysis, or interpretation of the data for the work; (2) participated in drafting of the work or revising it critically for important intellectual content; (3) approved the final version to be published; and (4) agreed to be accountable for all aspects of the work, ensuring that questions related to the accuracy or integrity of the work are appropriately investigated and resolved.

\section{References}

1 American Heart Association Web Pages; https://www.heart.org/en/health-topics/metabolic-syndrome/about-metabolic-syndrome Accessed January 8, 2021.

2 Boutayeb A, Boutayeb S, Boutayeb W. Multimorbidity of non communicable diseases and equity in WHO Eastern Mediterranean countries. Int J Equity Health. 2013;12:60.
3 De Carvalho Vidigal F, Bressan J, Babio N, Salas-Salvadó J. Prevalence of metabolic syndrome in Brazilian adults: a systematic review. BMC Public Health. 2013;13:1198.

$4 \mathrm{Fu}$ J, Prasad HC. Changing epidemiology of metabolic syndrome and type 2 diabetes in Chinese youth. Curr Diab Rep. 2014;14(1): 447.
5 Tune JD, Goodwill AG, Sassoon DJ, Mather KJ. Cardiovascular consequences of metabolic syndrome. Transl Res. 2017;183:57-70.

6 Barber M, Wright F, Stott DJ, Langhorne P Predictors of early neurological deterioration after ischaemic stroke: a case-control study. Gerontology. 2004;50(2):102-9. 
7 Boudreau DM, Malone DC, Raebel MA, Fishman PA, Nichols GA, Feldstein AC, et al. Health care utilization and costs by metabolic syndrome risk factors. Metab Syndr Relat Disord. 2009;7(4):305-14.

8 Halvorson BD, Whitehead SN, Mcguire JJ, Wiseman RW, Frisbee JC. Endothelium-dependent impairments to cerebral vascular reactivity with type 2 diabetes mellitus in the Goto-Kakizaki rat. AM J Physiol-Reg I. 2019; 317(1):R149-59.

9 Jarajapu YPR, Knot HJ. Relative contribution of Rho kinase and protein kinase $\mathrm{C}$ to myogenic tone in rat cerebral arteries in hypertension. Am J Physiol Hear Circ Physiol.. 2005; 289:H1917-22.

10 Pires PW, Dams Ramos CM, Matin N, Dorrance AM. The effects of hypertension on the cerebral circulation. Am J Physiol Heart Circ Physiol. 2013;304(12):H1598-614.

11 Lawes CM, Bennett DA, Feigin VL, Rodgers A. Blood pressure and stroke: an overview of published reviews. Stroke. 2004;35(4):102485.

12 Bray GA, York DA. Genetically transmitted obesity in rodents. Physiol Rev. 1971;51(3): 598-646.

13 Brooks SD, DeVallance E, d'Audiffret AC, Frisbee SJ, Tabone LE, Shrader CD, et al. Metabolic syndrome impairs reactivity and wall mechanics of cerebral resistance arteries in obese Zucker rats. Am J Physiol Heart Circ Physiol. 2015;309(11):H1846-59.

14 Frisbee JC. Hypertension-independent microvascular rarefaction in the obese zucker rat model of the metabolic syndrome. Microcirculation. 2005 Jul-Aug;12(5):383-92.
15 Aleixandre De Artiñano A, Miguel Castro M. Experimental rat models to study the metabolic syndrome. Br J Nutr. 2009;102(9):124653.

16 Lombard JH, Liu Y, Fredricks KT, Bizub DM, Roman RJ, Rusch NJ. Electrical and mechanical responses of rat middle cerebral arteries to reduced PO2 and prostacyclin. Am J Physiol. 1999;276(2 Pt 2):H509-16.

17 Phillips SA, Sylvester FA, Frisbee JC. Oxidant stress and constrictor reactivity impair cerebral artery dilation in obese Zucker rats. Am J Physiol Regul Integr Comp Physiol. 2005; 288(2):R522-30

18 Takenaka T, Suzuki H, Okada H, Hayashi K, Kanno Y, Saruta T. Mechanosensitive cation channels mediate afferent arteriolar myogenic constriction in the isolated rat kidney. J Physiol. 1998;511 ( Pt 1)(1):245-53.

19 Scotland RS, Chauhan S, Davis C, De Felipe C, Hunt S, Kabir J, et al. Vanilloid receptor TRPV1, sensory C-fibers, and vascular autoregulation: A novel mechanism involved in myogenic constriction. Circ Res. 2004;95(10): 1027-34.

20 Takenaka T, Suzuki H, Okada H, Hayashi K, Ozawa Y, Saruta T. Biophysical signals underlying myogenic responses in rat interlobular artery. Hypertension. 1998;32(6):1060-5.

21 Butcher JT, Goodwill AG, Stanley SC, Frisbee JC. Differential impact of dilator stimuli on increased myogenic activation of cerebral and skeletal muscle resistance arterioles in obese zucker rats. Microcirculation. 2013;20(7): $579-89$.
22 Chantler PD, Shrader CD, Tabone LE, d'Audiffret AC, Huseynova K, Brooks SD, et al. Cerebral cortical microvascular rarefaction in metabolic syndrome is dependent on insulin resistance and loss of nitric oxide bioavailability. Microcirculation. 2015;22(6):435-45.

23 Turturici M, Roatta S. Effects of gadolinium chloride on basal flow and compression-induced rapid hyperemia in the rabbit masseter muscle. J Physiol Pharmacol. 2014;65(3):40915.

24 Paravicini TM, Drummond GR, Sobey CG. Reactive oxygen species in the cerebral circulation: physiological roles and therapeutic implications for hypertension and stroke. Drugs. 2004;64(19):2143-57.

25 Muller-Delp JM, Gurovich AN, Christou DD, Leeuwenburgh C. Redox balance in the aging microcirculation: new friends, new foes, and new clinical directions. Microcirculation. 2012;19(1):19-28.

26 Gore RW. Wall stress: a determinant of regional differences in response of frog microvessels to norepinephrine. Am J Physiol. 1972;222(1):82-91.

27 McCarron JG, Lee MD, Wilson C. The endothelium solves problems that endothelial cells do not know exist. Trends Pharmacol Sci. 2017;38(4):322-38.

28 Radeva MY, Waschke J. Mind the gap: mechanisms regulating the endothelial barrier. Acta Physiol. 2018;222(1):1-20.

29 Potente M, Carmeliet P. The link between angiogenesis and endothelial metabolism. Annu Rev Physiol. 2017;79:43-66. 